\title{
A Variable Rolling SLIP Model for a Conceptual Leg Shape to Increase Robustness of Uncertain Velocity on Unknown Terrain
}

\author{
Adar Gaathon* and Amir Degani ${ }^{\dagger}$ \\ Technion-Israel Institute of Technology, Haifa, Israel. \\ *adarg@ campus.technion.ac.il, ${ }^{\dagger}$ adegani@technion.ac.il
}

\begin{abstract}
The use of simple control schemes with only a few basic sensors and no feedback allows improved stability when traversing unforeseen rough terrain by applying a single controller. Exploiting multiple controllers simultaneously can further improve robustness but is often mechanically hard to implement, especially when stiffness modulation is a controller. To overcome this limitation, we investigate and simulate a leg shape that applies variable leg stiffness and free-leg length. The leg shape couples the physical parameters with the leg angle of a monopod, while the leg orientation is governed with only a simple control law during the flight phase. We study the usage of an optimal controller coupling and show that it can increase robustness to perturbations in the initial horizontal velocity when traversing unknown rough terrain. This work presents the process of obtaining the optimal coupled parameters and demonstrates its benefits. This work also lays the foundations for a conceptual leg shape to exhibit the controllers physically.
\end{abstract} control

Keywords- legged locomotion; design optimization; minimalistic

\section{INTRODUCTION}

The ability of dynamic legged locomotion to traverse rough terrain is incomparable to the inherently limited ability of wheeled and tracked platforms [1]. Controlling dynamic legged robots is normally done using closed loop control, necessitating complex algorithms and fast and accurate sensors. What we term "minimalistic control schemes" require only little "live" low-frequency information about the terrain and the robot, usually with only apex and touchdown detection sensors. Such schemes are commonly used with the simple Spring Loaded Inverted Pendulum (SLIP) model [2], and govern a single controller, normally the leg angle relative to the ground. There are many examples of such control schemes, such as the Swing Leg Retraction (SLR) which controls the leg angle during decent by retracting the leg and helps to improve stability [3]. Other simple control methods can increase stability [4], keep the horizontal travel velocity constant, lower ground reaction forces [5] and increase efficiency [6].

Applying only one controller at each stride can prevent a premature failure, but exploiting multiple controllers at once may be beneficial to enlarge the reachable area of the robot; make the robot more resistant to controller errors; and handle controller limits, errors or other physical constraints [7]. Yet, modifying multiple controllers at once is not simple, since they are often hard to mechanically implement and control, may require an external power supply, or are not fast and accurate enough.
In this paper we study a variable version of the SLIP model to demonstrate that an optimal coupled combination of parameters can significantly increase robustness to unknown terrain level and initial conditions. We allow the free leg-length and leg stiffness to be modulated, albeit coupled with the leg angle. Specifically, we optimize the leg angle during descent and obtain the SLR and the continuous free leg-length and leg stiffness during stance. To do so, we formulate the equations of motion and optimization constraints and compare our results to an optimized ordinary SLIP model.

Our deeper intention is to find a physical embodiment of the optimal coupled parameters. We assume a leg shape contacting the ground can passively exert the desired physical parameters, namely, the stiffness and the free-leg length with only one actuator to govern the leg orientation during flight. During the stance phase, the actuator is disengaged. Using such a leg shape can improve the robustness of a robot to perturbations in both terrain and initial horizontal velocity without complicating the control scheme. An example of such a leg shape, that resembles RHex's legs [8], is described in Fig. 1, where four snapshots of a trajectory are depicted.

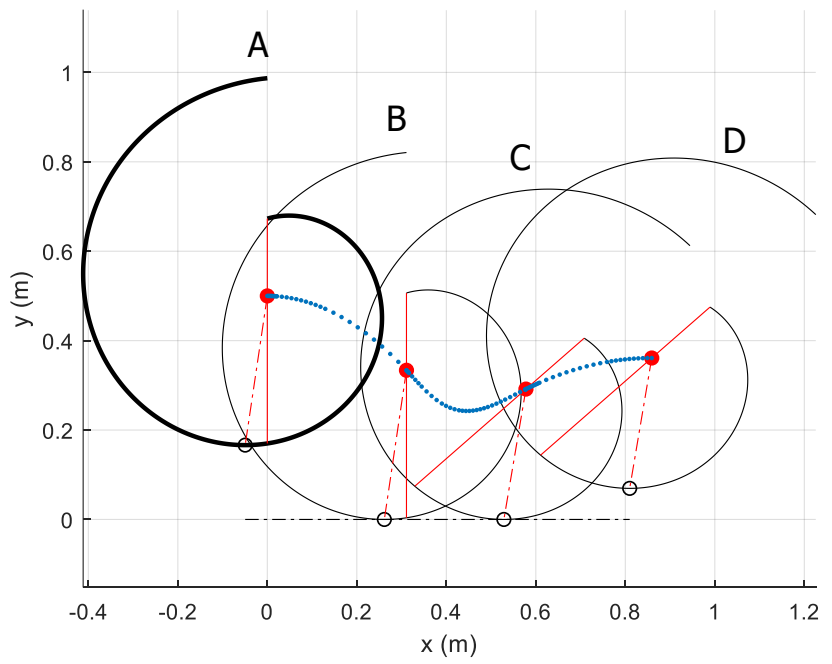

Fig. 1. An example of a leg shape and its rolling during stance in $(x, y)$ plane. The leg shape is denoted with a black curve; the hip, where the center mass is located, is denoted with a red dot; and the center of pressure (COP) is denoted with a black circle. The red continuous line represents the orientation. The dashed line denotes the vector of hip to COP. The blue dots denote the trajectory of the hip. Stages of a stride: A: (bold) starting at initial conditions; B: touchdown; C: liftoff; D: reaching apex. Note that the physical link of the hip to the leg shape is not presented here. 


\section{RELATED WORK}

\section{A. Minimalistic Control Methods}

Many legged robots are often modeled using the conservative planar Spring-Loaded Inverted Pendulum (SLIP) model [2] depicted in Fig. 2. Specific details about the SLIP model are given in Section III.

Many works suggested simple control for the SLIP model that use none or only a few simple sensors with no closed loop control strategy. In this paper we term such control methods minimalistic control schemes. The SLIP model can be selfstabilized using a fixed leg angle during descent on a flat terrain with no perturbation [9]. Swinging back the leg after reaching the apex (swing leg retraction, or SLR) increases the robustness of the system and copes with moderate unknown terrain variations [5], [10]-[14]. In SLR, the leg is brought to a predefined angle at apex and rotated during descent until touchdown.

Correctly tuning the SLR parameters can control the robot's height above preceding terrain level [10] and even keep a fixed relative height $\left(\mathrm{y}_{\text {rel }}\right)$, which better handles unforeseen up or down steps [13]. Conveniently, such modification of the control parameters is well described by a second-degree polynomial [15]. Keeping the relative height above ground when traversing rough terrain holds several advantages (some of which have been stated in [5]): 1) it enables the robot to perform more jumps on random terrain until it fails, as all steps have "best knowledge" to the upcoming terrain; 2) it induces the robot to run at maximum averaged horizontal velocity, since only part of the energy is expended as potential energy; 3) the descents are generally limited, lowering ground reaction forces (GRF). Importantly, maintaining an accurate relative height from unknown or poorly perceived rough ground is correlated to an improved ability to traverse longer distances until failure [13].

The effect of leg stiffness modification is also presented in the literature. Adapting the stiffness can better control the gait by tuning the natural frequency [16]. Humans adapt their leg stiffness to accommodate changes in terrain stiffness to maintain similar running mechanics [17]. A segmented leg couples stiffness with the leg angle and can enlarge the stability region as a result of its nonlinear stiffness during stance [18]. Several other works have shown that using a stiffness-tunable model can reduce ground reaction [19], control the running

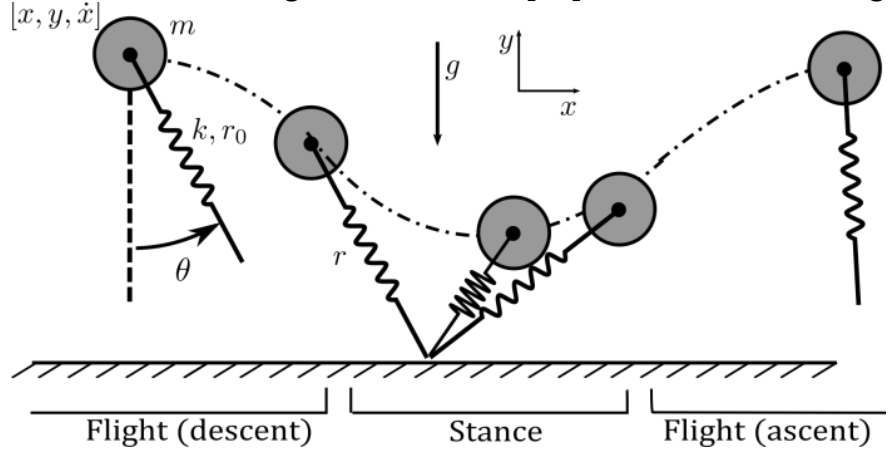

Fig. 2. The 2D SLIP model speed [20], and prevent premature failure (crashing) modes at the end of stance [7].

Mechanically designing robotic legs with variable stiffness has been attempted in running robots [21]. Traditionally, stiffness control in robotic mechanisms was done by adjusting the gains at individual joints motor level [22]. Another method, though not efficient, is to use nature's inspired antagonistic springs [23]. Galloway, Clark and Koditschek designed a tunable stiffness composite C-shaped leg [24], where sliding a rigid spine along the leg varies the composite moment of inertia and stiffness while maintaining the global shape of the foot. This mechanism was later improved by introducing a motor to online tune the stiffness [22], [25]. The latter work used the tunable stiffness as a shock absorber, for energy efficiency (harmonic with actuation), and for dynamic stability.

\section{B. Leg Shape}

Multiple works have studied leg shapes and their influence on a legged robot. Running with compliant curved legs can be more efficient and robust with a faster recovery from perturbations than running with compliant straight legs [6], [26]. Adamczyk, Collins and Kuo showed that increasing foot curvature generally diminished the energy cost of walking and had an optimal value when the curvature radius was $30 \%$ of the leg length [27]. They explained that a longer foot reduces the directional change in velocity and thus reduces the work in step-to-step transition [28].

Jun and Clark [6] mentioned that adapting leg parameters can be done passively with curved legs with no direct actuation because of their geometry and the involved leg circular motion for running. Assuming no rolling occurs during stance, they used the TD-TSL model with segmented legs and estimated the leg stiffness during stance using the PRB model for curved beams [29]. They mapped several combinations of the leg shape 'aspect ratio' and leg angles that meet their definition for robustness- to run 50 consecutive steps successfully on flat terrain. They found the suitable leg angles for robustness merely for limits of the range but not as a control scheme.

Our current paper suggests the concept of a theoretical leg design and leg angle control to apply simultaneous coupled parameters during stance. Our work resembles somewhat the work of Rodrigues and Mason [30], where they used spiral shaped fingers for invariant grasping, while we use such shapes for locomotion. Our hypothesis is that an optimized coupling of several physical parameters can reduce deviations from a desired $\mathrm{y}_{\text {rel, }}$ which induces robustness to unpredicted ground levels, when errors in the initial velocity arise. We use a modified version of the SLIP model with variable parameters, namely, spring (leg) rest-length, leg stiffness, and leg angle. We compare our simulation findings to the optimized constantparameter common SLIP model and analyze the results. We further study the physical embodiment of a leg shape including the effects of rolling during stance. The shape passively employs the optimal coupled parameters of stiffness and freeleg length during the stance phase. In our design, the leg is driven with only an orientation controller during the flight phase. 


\section{METHODS}

\section{A. Constant and Variable SLIP Models}

The SLIP model [2] (Fig. 2) comprises a point mass and a springy massless leg that eliminates impacts upon touchdown. The stride is divided into three recurring sections: flight (descent), stance, and flight (ascent). The stance equations are given by

$$
\left\{\begin{array}{c}
m \ddot{r}=m r \dot{\theta}^{2}+k\left(r_{0}-r\right)-m g \cos (\theta) \\
m r^{2} \ddot{\theta}=-2 m \dot{\theta} r \dot{r}+m g r \sin (\theta)
\end{array},\right.
$$

where $m$ is the mass, $g$ is the gravitational acceleration, $k$ is the constant leg stiffness and $r_{0}$ is the constant free-leg length. In this paper we use flight phase control (as opposed to the stance phase control), where we control the leg angle only during the relatively long descent period, allowing more time for control [14]. The leg angle actuator follows a temporal second-degree polynomial. During stance, the actuator is disengaged (for example by a mechanical clutch), which means the orientation of the leg is determined only by the model's dynamics.

In addition to the common SLIP model (what we term constant SLIP), we investigate a modified model, where we allow a modulation of the stiffness and leg-length during stance. Concretely, these parameters are dependent on the leg angle $\theta$ ( $k=k(\theta), r_{0}=r_{0}(\theta)$ ). The first coupled differential equation regarding $\ddot{r}$ of the so-called variable SLIP is identical to (1); yet, it should be noted that $k$ and $r_{0}$ are now dependent on $\theta$. Equation (2) is transformed into

$$
\begin{aligned}
& m r^{2} \ddot{\theta}= \\
& \quad-2 m \dot{\theta} r \dot{r}+m g r \sin (\theta)-\frac{d k}{d \theta} \frac{\left(r_{0}-r\right)^{2}}{2}-k \frac{d r_{0}}{d \theta}\left(r_{0}-r\right),
\end{aligned}
$$

where the last two terms in (3) are due to the variable stiffness and free-leg length. The derivation of the equations is outlined in Appendix A.

As opposed to the constant SLIP, the nominal properties of variable SLIP constantly change, making the favorable nondimensional analysis less intuitive for the reader, thus we will use throughout the work the dimensional analysis.

We expanded the second-degree leg angle polynomial to polynomials of stiffness and free-leg length with respect to leg angle. Specifically, $\theta(t)=\alpha_{1} t^{2}+\alpha_{2} t+\alpha_{3} ; r_{0}(\theta)=\beta_{1} \theta^{2}+$ $\beta_{2} \theta+\beta_{3} ; k(\theta)=\gamma_{1} \theta^{2}+\gamma_{2} \theta+\gamma_{3}$. The polynomial of $r_{0}$ describes a leg which can vary substantially between a helix to an ellipse. Assuming a polynomial behavior facilitates the derivatives written in the variable SLIP equations, but prevents us from using the varying parameter models in the literature, like the TD-HCL [6] and the R-SLIP [31], who are both circular.

\section{B. Rolling SLIP}

The variable SLIP model, presented in Section III.A, allows the stiffness and free leg-length to be modified as polynomials. We presume a leg shape, like the leg presented in Fig. 3, can passively exert these physical parameters when the leg rolls on the ground. Rolling is the continuous change of the ground contact point (often termed the center of pressure, or COP) along the leg circumference during stance and is denoted by the

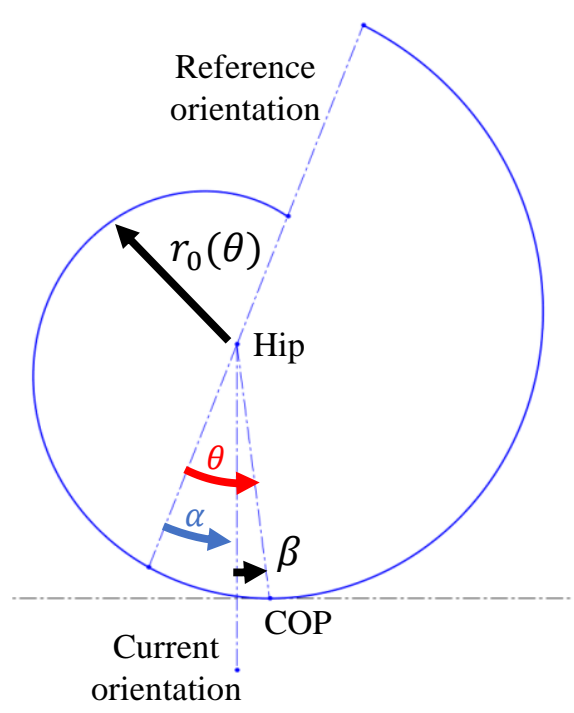

Fig. 3. An example of a polynomial leg shape and related definitions

angle $\theta$ in Fig. 3. The rotation axis is at the hip which is also the center of mass (COM). At each orientation, denoted by the angle $\alpha$, the leg touches the ground at an angle $\theta$ that depends on the shape. The angle of the COP relative to the current orientation is denoted by $\beta$, leading to the relation $\theta=\alpha+\beta$.

As in the variable SLIP model, we assume the free-leg length is of the form $r_{0}(\theta)=\beta_{1} \theta^{2}+\beta_{2} \theta+\beta_{3}$, and that the stiffness is $k(\theta)=\gamma_{1} \theta^{2}+\gamma_{2} \theta+\gamma_{3}$, where $-\pi \leq \theta \leq \pi$. In the present model, the orientation angle $(\alpha)$ does not equal the COP angle $(\theta)$. Thus, the hip actuator, that controls the leg orientation during descent, follows the polynomial control law $\alpha(t)=$ $\alpha_{1} t^{2}+\alpha_{2} t+\alpha_{3}$. As in the previous models, the actuator is disengaged during the stance phase.

The effect of rolling in itself was studies in several works, like in [6], [32], yet with no change in leg parameters. Our rolling SLIP model combines both rolling and the ability to change in mid-stance the leg's stiffness and free length due to its shape. Appendix B details the derivation of the rolling SLIP equations. Yet, the equations of motion themselves are not listed in this manuscript due to their length and complexity. The three models are summarized in Table 1.

\section{Optimization of the Polynomials of the Three Models}

We aim to reduce deviations of a one-stride apex height from a predetermined relative height above unknown ground level when perturbations in the initial horizontal velocity are encountered. Keeping a relative height is related with long term

\begin{tabular}{|l|c|c|c|}
\multicolumn{1}{|c}{ TABLE 1. SUMMARY OF INVESTIGATED SLIP MODELS } \\
\hline Model & \multicolumn{1}{|c|}{ Constant } & Variable & Rolling \\
\hline Description & $\begin{array}{c}\text { COP location does } \\
\text { not change during } \\
\text { stance; stiffness } \\
\text { and free-leg length } \\
\text { remain constant }\end{array}$ & $\begin{array}{c}\text { COP location does } \\
\text { not change during } \\
\text { stance; stiffness } \\
\text { and free-leg length } \\
\text { can modulate }\end{array}$ & $\begin{array}{c}\text { COP location } \\
\text { changes during } \\
\text { stance; stiffness } \\
\text { and free-leg length } \\
\text { can modulate }\end{array}$ \\
\hline $\begin{array}{l}\text { Number of } \\
\text { design } \\
\text { parameters }\end{array}$ & 5 & 9 & 9 \\
\hline
\end{tabular}


stability, which is our end goal. Our optimization process finds the optimal polynomials of the SLIP models; for the constant SLIP five coefficients which are $\alpha_{1}, \alpha_{2}, \alpha_{3}$ responsible for the leg angle rotation during flight and the constant stiffness and leg length; for the variable and rolling SLIP nine coefficients which are $\alpha_{1}, \alpha_{2}, \alpha_{3}, \beta_{1}, \beta_{2}, \beta_{3}, \gamma_{1}, \gamma_{2}, \gamma_{3}$. Simply put, the trajectory starts from an apex of a known height and unknown (but limited) horizontal velocity. On its descent, the leg angle (or orientation) is dictated according to the temporal polynomial of $\theta(t)$ (in constant and variable SLIP) and $\alpha(t)$ (in rolling SLIP). During stance, the stiffness and free-leg length are governed by polynomials of COP angle $(\theta)$.

The cost function is a double Euclidean norm of all deviations from $\mathrm{y}_{\mathrm{rel}}$. As mentioned earlier, keeping $\mathrm{y}_{\mathrm{rel}}$ was demonstrated to increase the long-term robustness. In mathematical form, the cost function for a relative height above terrain and initial height level is defined as

$$
\operatorname{cost}\left(y_{0}, y_{\text {rel }}\right)=\sqrt{\sum_{j=1}^{3}\left(\sqrt{\sum_{i=1}^{10}\left(\mathrm{y}_{\text {rel }(j)}-\left(y_{\text {apex }}-y_{\text {ter }_{(i)}}\right)\right)^{2}}\right)^{2}},
$$

where $\left(y_{\text {apex }}-y_{\text {ter }}^{i}\right)$ is the actual relative height above a tested ground level $y_{\text {ter }}=[-0.15,0.15] \mathrm{m}$ (equally spaced). The index $j$ is of the initial horizontal velocity vector $\dot{x}=$ $[0.3,0.5,0.7] \mathrm{m} / \mathrm{sec}$ that brackets our nominal velocity $0.5 \mathrm{~m} /$ sec. We repeated the optimization for various desired relative height above terrain, $y_{r e l}=[0.2,0.3, \ldots, 1.5] \mathrm{m}$; and for initial height levels $y_{0}=[0.5,0.6, \ldots, 1.5] \mathrm{m}$.

We applied the Nelder-Mead constrained optimization algorithm using MultiStart optimization of Matlab ${ }^{\mathrm{TM}}$ and repeated it (on average 3.4 times) to further validate our approach. We also analyzed the graphs of $\mathrm{f}_{\mathrm{val}}$, the minimum cost resulting from the optimization, to spot inconsistencies. The optimization is subjected to non-linear constraints that guarantee the parameters remain physically valid at touchdown, and that the legs are manufacturable. All trajectories that encounter non-physical (negative) stiffness or free length during stance were penalized and hence eliminated. Moreover, we limited the design parameters such that the evaluated leg angle is limited in the flight phase to reasonable values of $\left[-45^{\circ},+45^{\circ}\right]$ to prevent foretold failures. The free-leg length and stiffness were also bounded for the values of the leg angle during flight: $0 \leq r_{0} \leq 0.5 \mathrm{~m}, \forall-45^{\circ} \leq \theta \leq 45^{\circ}$ and $0 \leq$ $k \leq 10 \mathrm{kN} / \mathrm{m}, \forall-45^{\circ} \leq \theta \leq 45^{\circ}$. The parameters were not bounded during the stance phase, except for non-physical values, to prevent over-limiting. Additional constraints regarding the physical leg shape, relating the rolling SLIP model are listed in Appendix C.

\section{RESULTS AND DISCUSSION}

\section{A. Comparing Constant and Variable SLIP}

We performed the optimization process for each pair of $(p, q)$. The results with $f_{\text {val }}$ lower than 0.7 are depicted in Fig. 4. The $f_{\text {val }}$ of the variable SLIP is generally much lower than that of the constant SLIP. For both, the diagonal of $y_{0}=y_{r e l}$ is a borderline of similar values of $f_{v a l}$. Beyond this line, in the

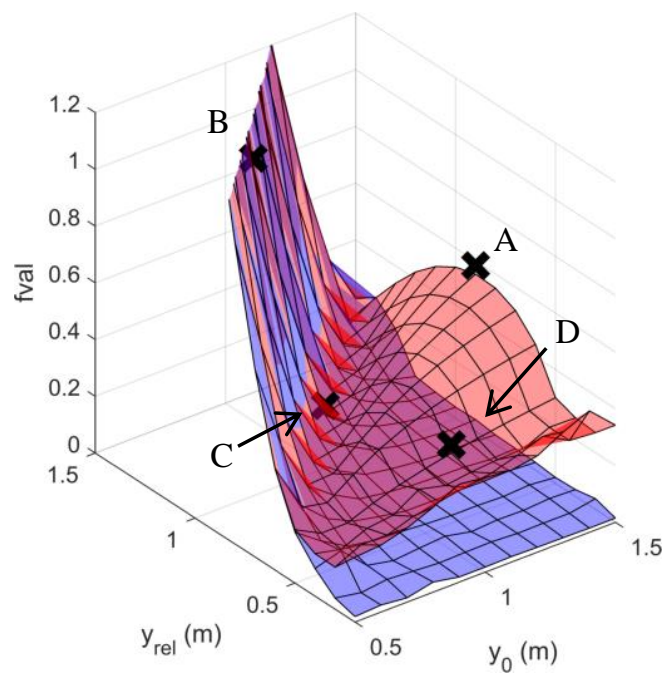

Fig. 4. Isometric overlayed view of $\mathrm{f}_{\mathrm{val}}$ for the constant (red) and variable (blue) SLIP in the $y_{0}, y_{\text {rel }}$ plane for unknown initial horizotnal velocity. Query points are marked with black crosses. Note the rapid elevation in $\mathrm{f}_{\mathrm{val}}$ in the region $y_{\text {rel }}>y_{0}$.

region where $y_{\text {rel }}>y_{0}, \mathrm{f}_{\mathrm{val}}$ of both constant and variable SLIP grows rapidly. This is due to an energy barrier, meaning that the trajectory cannot reach the desired relative height, because it is too high. To further investigate, we marked four query points $(\mathrm{A}, \mathrm{B}, \mathrm{C}, \mathrm{D})$ in characteristic spots in the $y_{0}, y_{\text {rel }}$ plane. Table 2 lists the $\mathrm{f}_{\text {val }}$ of both the constant and variable SLIP in the query points, and the relative improvement of the variable SLIP over the constant SLIP, which is over $92 \%$ in point A. The relative improvement formula is depicted in the bottom row of Table 2 .

If we assume the initial horizontal velocity is known, that is, the initial velocity vector is simply $0.5 \mathrm{~m} / \mathrm{sec}$, the performance of keeping a relative height above ground dramatically improves ( $\mathrm{f}_{\mathrm{val}}$ drops). Additionally, $\mathrm{f}_{\mathrm{val}}$ in both the constant and variable SLIP, is very similar (see Fig. 5). The findings can be partially explained in that the cost function considers only one instance of horizontal velocity, and not three, as in the previous case. The main reason, though, is that the constant SLIP easily performs the task of maintaining a relative height above terrain, and no significant, if any, improvement can be achieved by the variable SLIP.

\section{B. Parameters During Stance}

We examined the actual leg stiffness and free-leg length during stance. For the constant SLIP, the parameters remain constant throughout the stance. For the variable SLIP we simulated the stance phase and obtained the free-leg length and stiffness along the phase on all terrain levels and for all horizontal velocities. We then took the maximum of the parameters for each point in the $y_{0}, y_{\text {rel }}$ plane and overlaid them in Fig. 6. The constant SLIP tends to raise stiffness and leg length values to accomplish the task, while the variable SLIP generally keeps the maximum values much lower. The bulge in the free-leg length in the variable SLIP, seen in Fig. 6 and listed in Table 3, exceeds the maximum allowed value of $0.5 \mathrm{~m}$. This is because the non-linear constraints do not bound the values throughout the stance phase. We verified that the deviation from 


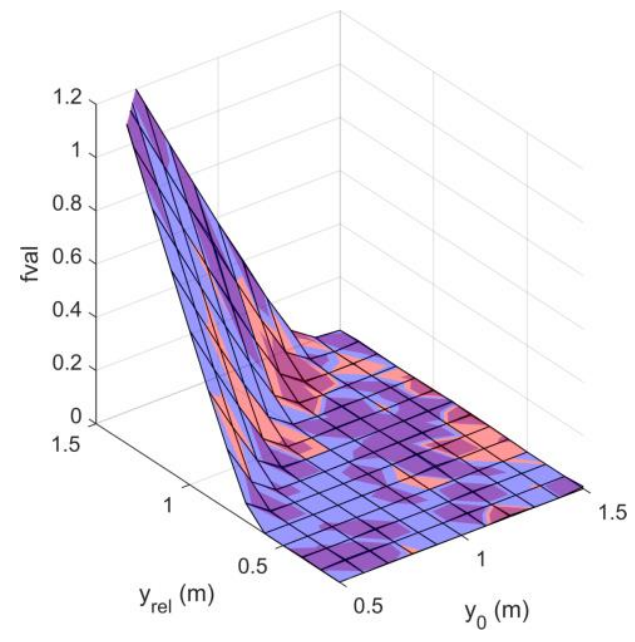

Fig. 5. Isometric overlayed view of the cost value for the constant (red) and variable (blue) SLIP in the $y_{0}, y_{\text {rel }}$ plane for a known initial horizontal velcoity.

TABLE 2. F $\mathrm{VAL}_{\mathrm{V}}$ OF CHARACTERISTIC QUERY POINTS IN THE $y_{0}, y_{r e l}$ PLANE

\begin{tabular}{|l|c|c|c|c|}
\hline & \multicolumn{4}{|c|}{$\mathbf{f}_{\text {val }}$} \\
\hline \multicolumn{1}{|c|}{ Point } & $\boldsymbol{A}$ & $\boldsymbol{B}$ & $\boldsymbol{C}$ & $\boldsymbol{D}$ \\
\hline Location $\left[y_{0}, y_{\text {rel }}\right]$ & $(1.5,0.9)$ & $(0.8,1.1)$ & $(1,1)$ & $(1.1,0.5)$ \\
\hline Constant SLIP & 0.554 & 1.086 & 0.190 & 0.247 \\
\hline Variable SLIP & 0.041 & 1.066 & 0.185 & 0.027 \\
\hline $\begin{array}{l}\text { Improvement } \\
\left(\frac{\mathrm{f}_{\text {val }}(\text { const })-\mathrm{f}_{\text {val }}(\text { var })}{\mathrm{f}_{\text {val }}(\text { const })}\right)(\%)\end{array}$ & 92.60 & 1.84 & 2.63 & 89.07 \\
\hline
\end{tabular}

the maximum value does not give a significant advantage to the variable SLIP over the constant SLIP. Table 3 lists the parameters in the four query points as well as the minimum, mean and maximum parameters in the $y_{0}, y_{\text {rel }}$ plane during stance. Note that the mean stiffness of the variable SLIP in the $y_{0}, y_{\text {rel }}$ plane is much lower than that of the constant SLIP $\operatorname{model}(3.422 \mathrm{kN} / \mathrm{m}$ vs $8.976 \mathrm{kN} / \mathrm{m})$. The same occurs with the free-leg length that is lower in the variable SLIP $(0.281 \mathrm{~m}$ vs $0.454 \mathrm{~m}$ ). The reason for these large differences may be attributed to the fact that the constant SLIP has fewer degrees of freedom; it has only one value of stiffness and leg length for all terrain levels and all initial horizontal velocities. Higher values of stiffness and leg length may not necessarily lead to an accurate relative height, but they are needed to prevent the body from colliding with the ground, which is severely penalized.

\section{Sensitivity Analysis}

We inspected the sensitivity of $f_{\text {val }}$ to perturbations in the controller values. We changed the touchdown leg angle by one degree with respect to the nominal expected value from the optimization process (i.e., $\theta_{\text {pert }}(t)=\alpha_{1 \text { opt }} t^{2}+\alpha_{2 o p t} t+$ $\alpha_{3 o p t}+1 \mathrm{deg}$ ), which is a typical experimental setup tolerance. Fig. 7 shows that in general, the variable SLIP is more sensitive to perturbations in the controllers compared to the constant SLIP. This may be because the variable SLIP is tailored to reject perturbation in initial horizontal velocity and not in other parameters. In other words, there is a tradeoff in the robustness. We proceeded and measured the change of the $f_{v a l}$ when perturbing the stiffness by $300 \mathrm{~N} / \mathrm{m}$ and the leg length by $0.01 \mathrm{~m}$ (each perturbation by itself). Table 4 lists the nominal
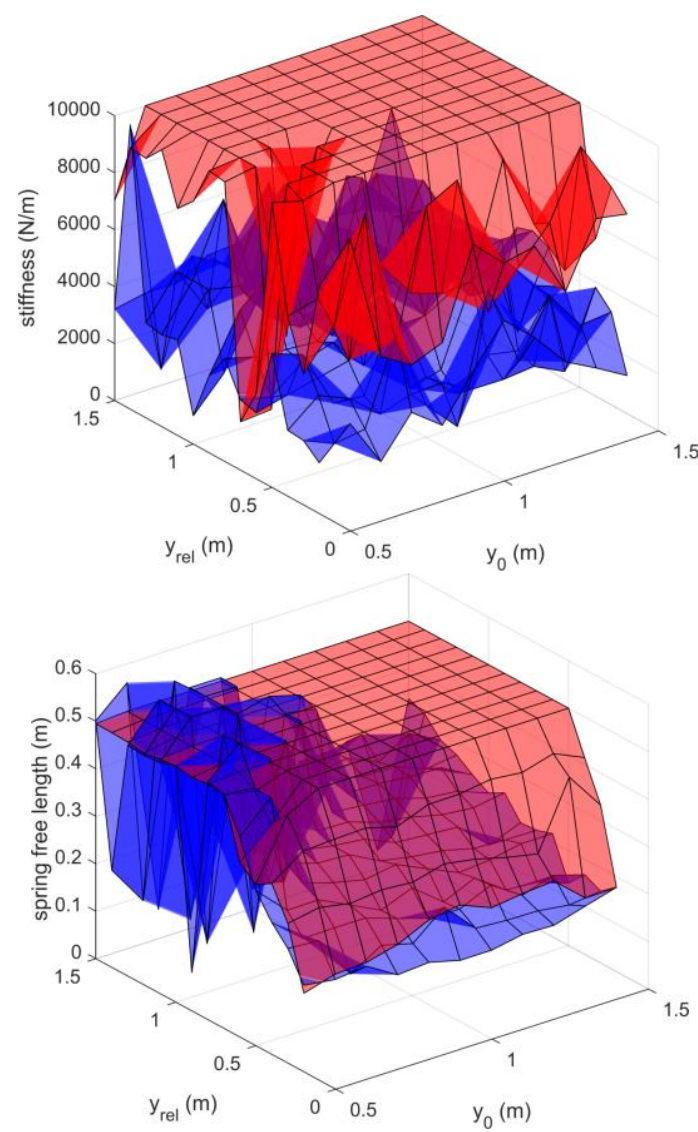

Fig. 6. Optimal parameters (maximum stiffness- top figure; maximum leg length- bottom figure) of the constant (red) and variable (blue) SLIP. Values are presented in the $y_{0}, y_{\text {rel }}$ plane.

TABLE 3. MAXIMUM PARAMETERS OF THE CONSTANT AND VARIABLE SLIP DURING THE STANCE PHASE

\begin{tabular}{|c|c|c|c|c|c|c|c|c|}
\hline & & \multicolumn{6}{|c|}{ Leg length and Leg Stiffness } \\
\hline & & \multicolumn{4}{c|}{ Point } & \multicolumn{3}{c|}{$\mathbf{y}_{\mathbf{0}}, \mathbf{y}_{\text {rel }}$ plane } \\
\hline & Model & $\boldsymbol{A}$ & $\boldsymbol{B}$ & $\boldsymbol{C}$ & $\boldsymbol{D}$ & Min & Mean & Max \\
\hline \multirow{2}{*}{$\begin{array}{c}\text { Stiffness } \\
(\mathrm{kN} / \mathrm{m})\end{array}$} & Constant & 10 & 10 & 10 & 10 & 1.698 & 8.976 & 10 \\
\cline { 2 - 10 } & Variable & 4.390 & 3.078 & 2.908 & 1.180 & 0.993 & 3.422 & 9.994 \\
\hline \multirow{2}{*}{$r_{0}(\mathrm{~m})$} & Constant & 0.5 & 0.5 & 0.5 & 0.5 & 0.165 & 0.454 & 0.5 \\
\cline { 2 - 9 } & Variable & 0.248 & 0.065 & 0.294 & 0.176 & 0.038 & 0.281 & 0.561 \\
\hline
\end{tabular}

value of both the constant and variable SLIP (no parameter perturbation); the $f_{\text {val }}$ for the perturbation of each parameter; and the relative improvement of the variable SLIP over the constant SLIP. In points $\mathrm{A}$ and $\mathrm{D}$, the variable SLIP $\mathrm{f}_{\mathrm{val}}$ is significantly favorable compared to the constant SLIP, although with lower significance compared to the nominal values. In points B and $\mathrm{C}$, which are situated on the energy barrier and beyond it, the variable SLIP is inferior in perturbations in the free-leg length and stiffness compared to the constant SLIP. It should be noted that the nominal $f_{\text {val }}$ of both schemes in points $B$ and $C$ was very similar, meaning that these points are the boundary of the valuable range of using the variable SLIP over the constant SLIP.

\section{Unknown Initial Height}

Previous sections have assumed the initial height is known and have found the optimal coupling of stiffness and free-leg length to reach a relative height above ground for each initial 
height. This section assumes the initial height is unknown. We wish to find the optimal polynomials of the leg angle, stiffness and free length that allow reaching a relative height above ground while handling a noise in horizontal velocity and unknown initial height (within bounds). We compared the performance of the optimized variable and constant SLIP. The cost function is now a bit simpler

$$
\operatorname{cost}\left(y_{\text {rel }}\right)=\sqrt{\sum_{j=1}^{3}\left(\sum_{i=1}^{11} \sqrt{\left(y_{\text {rel }_{(i)}}-y_{\text {apex }}\right)^{2}}\right)^{2}} .
$$

Here, the index $i$ relates to the initial height above ground $y_{0}=[0.5,1.5] \mathrm{m}$, and $j$ remains the index of the initial horizontal velocity. Since the initial height is changed during the optimization, the terrain level expression is omitted. Furthermore, the optimization is repeated only for the desired relative height vector.

After obtaining the optimal polynomial coefficients we used the polynomials to test the performance on variable terrain levels from various known initial heights and velocities.

Table 5 lists the $f_{\text {val }}$ results. Naturally, when using polynomials obtained by an optimization with an unknown initial height, the $f_{\text {val }}$ is higher in both SLIP models (relative to a known initial height). Yet, the rise in $f_{\text {val }}$ is much more significant in the variable SLIP than in the constant SLIP. Also,
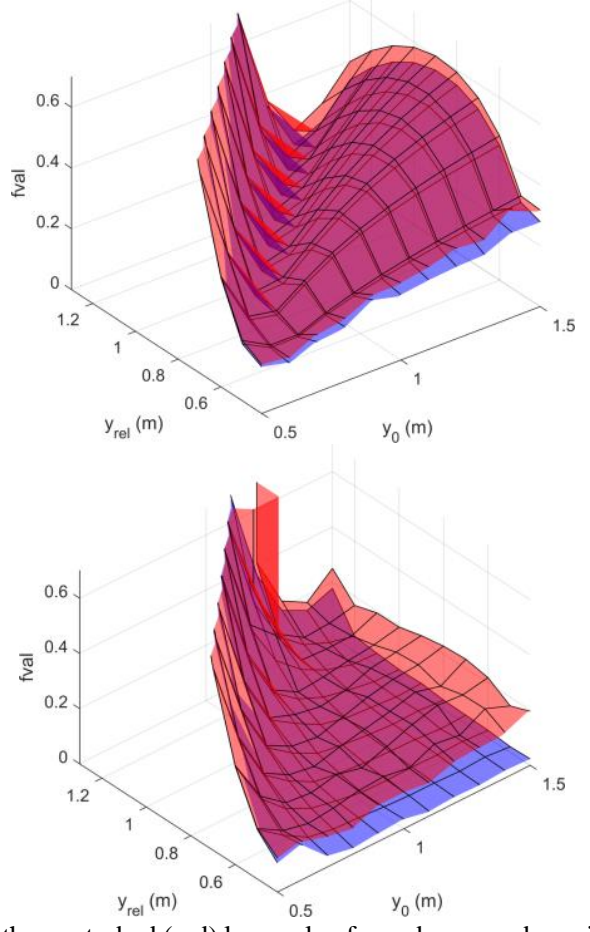

Fig. 7. $\mathrm{f}_{\text {val }}$ with a perturbed (red) leg angle of one degree and nominal value (blue) for the constant (top) and variable (bottom) SLIP for uknown intial horizontal velocities.
TABLE 4. F $\mathrm{F}_{\mathrm{VAL}}$ OF PERTURBATION OF LEG ANGLE $(\theta)$; STIFFNESS $(k)$; FREE-LEG LENGTH $\left(r_{0}\right)$; AND THE RATE OF IMPROVEMENT OF THE VARIABLE SLIP RELATIVE TO THE CONSTANT SLIP

\begin{tabular}{|l|l|c|c|c|c|}
\hline & \multicolumn{5}{|c|}{$\mathbf{f}_{\text {val }}$} \\
\hline \multirow{3}{*}{$\begin{array}{c}\text { Perturbation } \\
\text { No }\end{array}$} & Model/Point & $\boldsymbol{A}$ & $\boldsymbol{B}$ & $\boldsymbol{C}$ & $\boldsymbol{D}$ \\
\hline \multirow{4}{*}{$\theta$} & Constant & 0.554 & 1.086 & 0.190 & 0.247 \\
\cline { 2 - 6 } & Variable & 0.041 & 1.066 & 0.185 & 0.027 \\
\cline { 2 - 6 } & Imprv (\%) & $\mathbf{9 2 . 6 0}$ & $\mathbf{1 . 8 4}$ & $\mathbf{2 . 6 3}$ & $\mathbf{8 9 . 0 7}$ \\
\hline \multirow{4}{*}{$k$} & Constant & 0.601 & 1.089 & 0.221 & 0.261 \\
\cline { 2 - 6 } & Variable & 0.214 & 1.076 & 0.194 & 0.127 \\
\cline { 2 - 6 } & Imprv (\%) & $\mathbf{6 4 . 3 9}$ & $\mathbf{1 . 1 9}$ & $\mathbf{1 2 . 2 2}$ & $\mathbf{5 1 . 3 4}$ \\
\hline \multirow{3}{*}{$r_{0}$} & Constant & 0.552 & 1.086 & 0.189 & 0.246 \\
\cline { 2 - 6 } & Variable & 0.455 & 1.089 & 0.194 & 0.098 \\
\cline { 2 - 6 } & Imprv (\%) & $\mathbf{1 7 . 5 7}$ & $\mathbf{- 0 . 2 8}$ & $\mathbf{- 2 . 6 5}$ & $\mathbf{6 0 . 1 6}$ \\
\cline { 2 - 6 } & Constant & 0.553 & 1.085 & 0.197 & 0.233 \\
\cline { 2 - 6 } & Variable & 0.145 & 1.457 & 0.234 & 0.132 \\
\cline { 2 - 6 } & Imprv (\%) & $\mathbf{7 3 . 7 8}$ & $\mathbf{- 3 4 . 2 9}$ & $\mathbf{- 1 8 . 7 8}$ & $\mathbf{4 3 . 3 5}$ \\
\hline
\end{tabular}

TABLE 5. F FAL OF KNOWN AND UNKNOWN INITIAL HEIGHT FOR THE CONSTANT

\begin{tabular}{|c|l|l|l|l|l|}
\hline & & \multicolumn{1}{c|}{$\mathbf{f}_{\text {val }}$} \\
\hline $\begin{array}{c}\text { Initial } \\
\text { height }\end{array}$ & Model/Point & $\boldsymbol{A}$ & $\boldsymbol{B}$ & $\boldsymbol{C}$ & $\boldsymbol{D}$ \\
\hline \multirow{3}{*}{ Known } & Constant & 0.554 & 1.086 & 0.190 & 0.247 \\
\cline { 2 - 6 } & Variable & 0.041 & 1.066 & 0.185 & 0.027 \\
\cline { 2 - 6 } & Imprv (\%) & $\mathbf{9 2 . 6 0}$ & $\mathbf{1 . 8 4}$ & $\mathbf{2 . 6 3}$ & $\mathbf{8 9 . 0 7}$ \\
\hline \multirow{3}{*}{ Unknown } & Constant & 0.556 & 1.099 & 0.208 & 0.260 \\
\cline { 2 - 6 } & Variable & 0.238 & 1.353 & 0.258 & 0.099 \\
\cline { 2 - 6 } & Imprv (\%) & $\mathbf{5 7 . 1 9}$ & $\mathbf{- 2 3 . 1 1}$ & $\mathbf{- 2 4 . 0 3}$ & $\mathbf{6 1 . 9 2}$ \\
\hline
\end{tabular}

in points $\mathrm{B}$ and $\mathrm{C}$, the variable SLIP $\mathrm{f}_{\mathrm{val}}$ is higher relative to the constant SLIP. Yet, observing points A and D, the variable SLIP is much better than the constant SLIP.

Examining Fig. 5, Fig. 6, and Fig. 7 allows a more comprehensive analysis. Relating to Fig. $5, \mathrm{f}_{\mathrm{val}}$ for both variable and constant SLIP greatly increase in the region $y_{r e l}>y_{0}$, probably due to an energy limit. Additionally, the $f_{\text {val }}$ values become very similar, leading to the thought that the variable SLIP polynomials are the same as those of the constant SLIP. Yet, the diverse actual parameters during stance in both SLIP models, depicted in Fig. 6 prove that the polynomials of both SLIP models are distinct. Due to the energy barrier, the cost of various polynomials is very similar. In other words, the polynomials are "equally bad". Table 5 and Fig. 7 support this assumption; point $\mathrm{B}$, which is beyond the energy barrier is the least affected by a leg angle perturbation.

\section{E. Comparing the Three Models}

Depicted in Fig. 8 are $\mathrm{f}_{\text {val }}$ of all three SLIP model: constant, variable and rolling. It is evident that the $\mathrm{f}_{\mathrm{val}}$ of the rolling model is significantly lower than that of the constant SLIP. Yet, the non-rolling variable $\mathrm{f}_{\mathrm{val}}$ is better. This outcome can be explained that the rolling model is a constrained version of the variable SLIP model. In both models nine parameters are subjected to optimization, yet rolling lowers the allowable design parameter space.

Each node in the $y_{0}, y_{\text {rel }}$ plane in Fig. 8 comprises a set of the optimal parameters, of which three dictate the leg shape. 


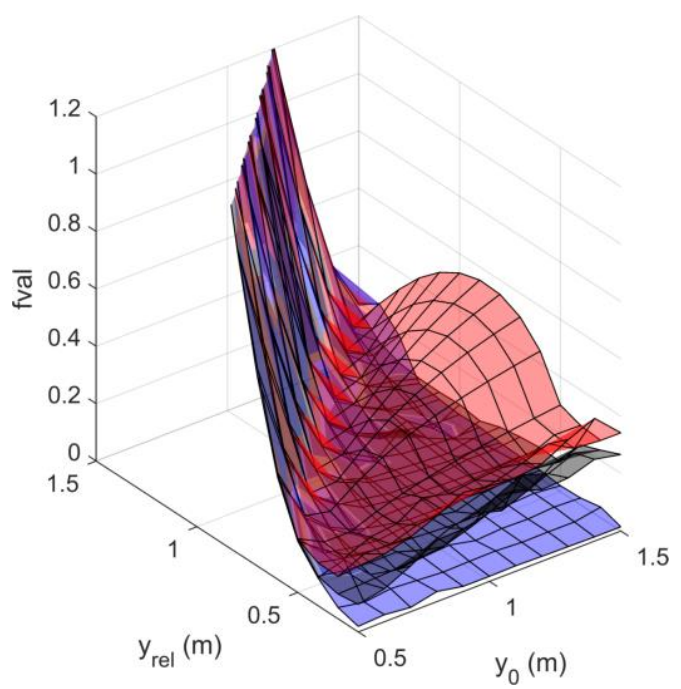

Fig. 8. Isometric overlayed view of $\mathrm{f}_{\mathrm{val}}$ for the constant (red), variable (blue) and rolling (black) SLIP in the $y_{0}, y_{\text {rel }}$ plane.

Plotting all the overlaid leg shapes as depicted in Fig. 9, reveals that they are roughly similar and that they are all left handed, meaning that the opening (if exists) is located on the left. No constraints where applied on the direction of the leg, meaning that a left-handed shape is favorable in minimizing the cost value.

\section{CONCLUSIONS AND FUTURE WORK}

This paper dealt with two main questions. The first is whether an optimal coupling of the leg parameters with leg angle controller will improve the ability to reach a relative height above ground, an important feature to increase stability of legged robots. The second topic of this paper laid the basics of a conceptual leg shape and its application in employing physical parameters upon ground contact.

Dealing with the variable SLIP model, we speculated that an optimal coupling would increase robustness to unpredicted changes in the initial horizontal velocity when traversing unknown rough terrain and proved our assumption. The variable SLIP greatly improves - up to $92 \%$ at the points tested - the robustness to perturbations in the horizontal velocity compared with the constant SLIP. The variable SLIP also uses much lower free-leg length (mean: $0.281 \mathrm{~m}$ vs 0.454 $\mathrm{m}$ ) and stiffness (mean: $3.422 \mathrm{kN} / \mathrm{m}$ vs $8.976 \mathrm{kN} / \mathrm{m}$ ), allowing a more compliant leg design. The variable SLIP does experience higher sensitivity to parameter perturbations and higher cost when the initial height is unknown relative to the constant SLIP. Yet, this behavior is in areas where $\mathrm{f}_{\mathrm{val}}$ is similar to that of the constant SLIP. The variable SLIP compensates by keeping higher robustness in the working areas (up to $74 \%$ ) for parameter perturbations, even when the initial height before descent is unknown (up to $62 \%$ ).

This paper additionally presented the foundations for a conceptual leg shape to support our hypothesis. We derived the rolling SLIP model that incorporates a rolling contact and manifests the ability to change physical parameters in mid stance, as the contact point progresses. We demonstrated that such a leg shape can substantially improve the robustness to unpredicted terrain levels and initial velocity.

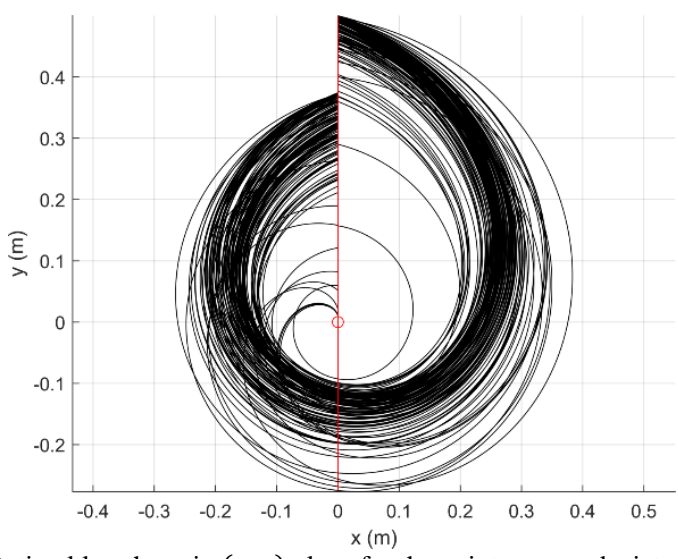

Fig. 9. Optimal leg shape in $(x, y)$ plane for the points $y_{0} y_{\text {rel }}$ depicted in Fig. 8 The hip is located in location $(x, y)=(0,0)$

Our intention is to implement this work and to create a physical leg shape that will combine the leg angle with stiffness and free-leg length to manifest the optimal polynomials we obtained. We will use a multibody simulator such as MSC Adams $^{\mathrm{TM}}$ and adjust some of the leg shape parameters such as cross-section, width and density. We will produce the leg with simple means like 3D printing and test it in real-life conditions.

\section{APPENDICES}

\section{A. Appendix A: Formulation of the Lagrangian for the Variable SLIP Model}

Starting with a kinematic analysis, we construct the Lagrangian of the hip (COM): $L=T-V$. The kinetic energy is

$$
T=\frac{\left(\dot{r}^{2}+r^{2} \dot{\theta}^{2}\right) m}{2} .
$$

The potential energy expression contains the expressions for stiffness and free-leg length that depend on the leg angle $\theta$.

$$
V=\frac{k(\theta)}{2}\left(r_{0}(\theta)-r(t)\right)^{2}+m g r \cos (\theta) .
$$

The derivations of the Lagrangian yields the terms $\frac{d k}{d \theta}$ and $\frac{d r_{0}}{d \theta}$ depicted in (3).

\section{B. Appendix B: Formulation of the Equations of Motion for the Rolling SLIP Model}

Following the description in Fig. 3, the location of the hip $(\mathrm{COM})$ relative to the touchdown location of the leg in the onset of the stance, i.e., the touchdown, $\left(x_{\text {foot }(t d)}, y_{\text {foot }(t d)}\right)$ can be written as

$$
\begin{gathered}
x_{\text {hip }}=x_{\text {foot }(t d)}-r \sin (\beta)+\int_{t_{t d}}^{t_{l o}} d s_{x y} \\
y_{\text {hip }}=y_{\text {foot }(t d)}+r \cos (\beta) .
\end{gathered}
$$

Note that $\left(x_{\text {foot }(t d)}, y_{\text {foot }(t d)}\right)$ is the location of the lowest point on the leg at touchdown. The integral term stands for the distance travelled during the stance in $(x, y)$ coordinates. In more details, $d s_{\mathrm{x} y}=\sqrt{d x^{2}+d y^{2}}$ is the infinitesimal distance along the curve of the leg shape in $(x, y)$ plane. We convert this term to polar coordinates, since we will use them in the 
formulation of the stance phase equations. We use the location of the hip relative to the current point of contact with the ground $\left(x_{\text {hip }}, y_{\text {hip }}\right)=\left(-r_{0} \cos (\theta), r_{0} \sin (\theta)\right)$, and the derivatives with respect to $\theta$ to obtain the useful term

$$
d s_{x y}=\sqrt{r_{0}^{\prime}(\theta)^{2}+r_{0}(\theta)^{2}} d \theta .
$$

Equation (8) is transformed to

$$
x_{\text {hip }}=x_{f o o t(t d)}-r \sin (\theta)+\int_{\theta_{t d}}^{\theta} \sqrt{r_{0}^{\prime}(\theta)^{2}+r_{0}(\theta)^{2}} d \theta \text {, }
$$

while (9) remains unchanged. Note that the travel on the ground considers only the nominal leg circumference $\left(d s=f\left(r_{0}\right)\right)$ without accounting for the compression of the leg shape, since the total circumference length is preserved.

For creating the Lagrangian, we derive the location of the hip with respect to time using the fundamental theorem of calculus and obtain the velocity of the hip

$\dot{x}_{\text {hip }}=-\dot{r} \sin (\beta)-r \dot{\beta} \cos (\beta)+\sqrt{r_{0}^{\prime}(\theta)^{2}+r_{0}(\theta)^{2}} \dot{\theta}$

$\dot{y}_{\text {hip }}=\dot{r} \cos (\beta)-r \dot{\beta} \sin (\beta)$.

The state variables are $r$ and $\theta$ and their derivatives. As such, $\beta$ and $\dot{\beta}$ are to be represented in terms of $\theta$ and $\dot{\theta}$. To do so, we write the expression for the vertical coordinate of the COP relative to the hip

$$
C O P_{y}=-r_{0}(\theta) \cos (\beta) .
$$

The touchdown angle $\beta$ can be found by finding the minimum of the leg location at y coordinate, i.e., by solving the equation

$$
\frac{d C O P_{y}}{d \beta}=-r_{0}^{\prime}(\theta) \cdot \frac{d \theta}{d \beta} \cos (\beta)+r_{0}(\theta) \sin (\beta)=0,
$$

where $r_{0}^{\prime}(\theta)=d r_{0} / d \theta$. Since $\theta=\alpha+\beta, \frac{d \theta}{d \beta}=1$. Thus,

$$
\frac{d C O P_{y}}{d \beta}=-r_{0}^{\prime}(\theta) \cos (\beta)+r_{0}(\theta) \sin (\beta) \text {. }
$$

Equating (16) to zero yields

$$
\beta=\operatorname{atan}\left(r_{0}^{\prime}(\theta) / r_{0}(\theta)\right) .
$$

As $\beta=f(\theta), \dot{\beta}=\dot{\theta} \cdot \beta^{\prime}$, which leads to

$$
\dot{\beta}=\dot{\theta} \cdot 1 /\left(1+\left(r_{0}^{\prime}(\theta) / r_{0}(\theta)\right)^{2}\right) \cdot \frac{d\left(r_{0}^{\prime}(\theta) / r_{0}(\theta)\right)}{d \theta} .
$$

Substituting (17) and (18) into (12) and (13), we can now formulate the Lagrangian with the state variables only. We state the kinematic and potential energies. The kinetic energy is

$$
T=\frac{\left(\dot{x}_{\text {hip }}^{2}+\dot{y}_{\text {hip }}{ }^{2}\right) m}{2} \text {. }
$$

The potential energy expression is the same as (7) (see Appendix A).

The equations of motion for the rolling SLIP are too combersome to fit within the paper and do not offer intuition either and are thus omitted.

\section{Appendix C: Leg Shape Constraints}

The use of a leg shape imposes additional constraints on the optimization process detailed in the Section III. Depicted in Fig. 10 are an example of a valid leg shape, where the leg's circumference allows a smooth terrain contact; and an invalid
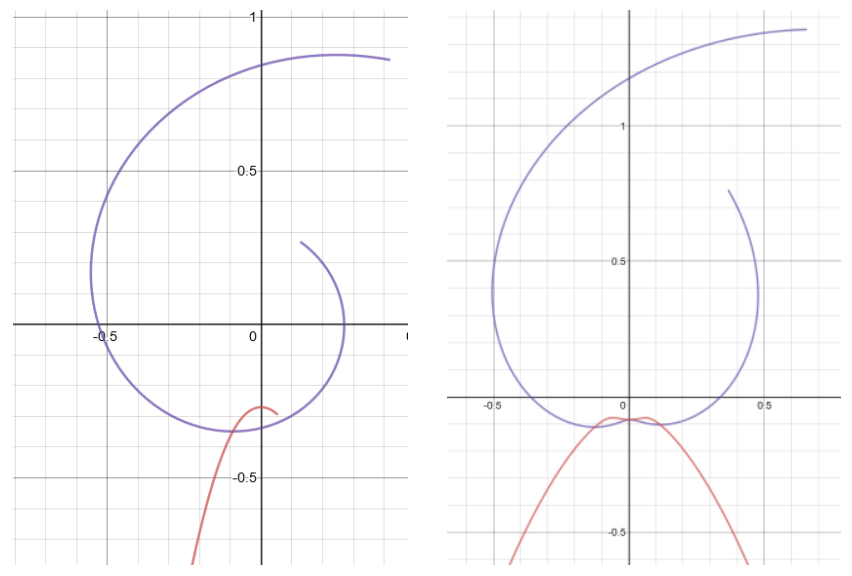

Fig. 10. Examples of a valid leg shape (left) and invalid leg shape (right). The leg shapes are denoted with a purple curve. The lowest point on the leg follows the red curve. The intersection of the purple and red curves is the lowest point on the leg for the current orientation.

leg shape with a cusp that will cause contact discontinuity while rolling. The red curve in Fig. 10 describes the lowest point on the leg and follows the parametric curve

$$
\begin{gathered}
x=r_{0} \sin (\beta) \\
y=-r_{0} \cos (\beta) .
\end{gathered}
$$

To prevent a cusp in the leg shape, the parametric curve must have only one maximum at most in the rotation region. Mathematically, we demand that $d^{2} y / d x^{2}<0$. Solving this inequality symbolically is very hard, if not impossible, so we used the Monte Carlo sampling method and manually defined constraining conditions on the leg shape polynomial.

\section{REFERENCES}

[1] M. H. Raibert, Legged robots that balance. The MIT Press,Cambridge, MA, 1986.

[2] R. Blickhan, "The spring-mass model for running and hopping.," $J$. Biomech., vol. 22, no. 11-12, pp. 1217-1227, 1989.

[3] A. Seyfarth, "Swing-leg retraction: a simple control model for stable running," J. Exp. Biol., vol. 206, no. 15, pp. 2547-2555, 2003.

[4] S. Riese and A. Seyfarth, "Stance leg control: variation of leg parameters supports stable hopping," Bioinspir. Biomim., vol. 7, no. 1, p. 016006, 2012

[5] J. G. D. Karssen, M. Haberland, M. Wisse, and S. Kim, "The optimal swing-leg retraction rate for running," in Proceedings of the IEEE International Conference on Robotics and Automation, 2011, pp. 40004006.

[6] J. Y. Jun and J. E. Clark, "Characterization of running with compliant curved legs," Bioinspir. Biomim., vol. 10, no. 4, p. 046008, 2015.

[7] M. Ernst, H. Geyer, and R. Blickhan, "Extension and customization of self-stability control in compliant legged systems," in Bioinspiration \& Biomimetics, 2012, vol. 7, no. 4, p. 046002.

[8] U. Saranli, M. Buehler, and D. E. Koditschek, "RHex: A Simple and Highly Mobile Robot," Int. J. Rob. Res., vol. 20, no. 7, pp. 616-631, 2001.

[9] A. Seyfarth, H. Geyer, M. Günther, and R. Blickhan, "A movement criterion for running," J. Biomech., vol. 35, no. 5, pp. 649-655, 2002.

[10] A. Seyfarth and H. Geyer, "Natural control of springlike running: Optimized self-stabilization," in Proceedings of the International Conference on Climbing and Walking Robots and the Support Technologies for Mobile Machines, 2002, no. 1, pp. 81-85.

[11] A. Seyfarth, G. Hartmut, and H. Hugh, "Swing-leg retraction: a simple control model for stable running," J. Exp. Biol., vol. 206, no. 15, pp. 2547$2555,2003$. 
[12] Y. Blum, S. W. Lipfert, J. Rummel, and A. Seyfarth, "Swing leg control in human Running," Bioinspir. Biomim., vol. 5, no. 2, pp. 1-11, 2010.

[13] N. Shemer and A. Degani, "A flight-phase terrain following control strategy for stable and robust hopping of a one-legged robot under large terrain variations," Bioinspir. Biomim., vol. 12, no. 4, 2017.

[14] H. R. Vejdani, Y. Blum, M. A. Daley, and J. W. Hurst, "Bio-inspired swing leg control for spring-mass robots running on ground with unexpected height disturbance.," Bioinspir. Biomim., vol. 8, no. 4, p. 046006, 2013.

[15] A. Gaathon and A. Degani, "Adaptive swing leg retraction control for robust dynamic locomotion under large terrain variations," in Proceedings of the International Conference on Climbing and Walking Robots and the Support Technologies for Mobile Machines, 2016, pp. 369-377.

[16] X. Zhou and S. Bi, "A survey of bio-inspired compliant legged robot designs," Bioinspir. Biomim., vol. 7, no. 4, p. 041001, 2012.

[17] D. P. Ferris, M. Louie, and C. T. Farley, "Running in the real world: adjusting leg stiffness for different surfaces," Proc. R. Soc. B Biol. Sci., vol. 265, no. 1400, pp. 989-994, 1998.

[18] J. Rummel and A. Seyfarth, "Stable Running with Segmented Legs," Int. J. Rob. Res., vol. 27, no. 8, pp. 919-934, 2008.

[19] J. Y. Jun and J. E. Clark, "Dynamic stability of variable stiffness running," in IEEE International Conference on Robotics and Automation (ICRA), 2009, pp. 1756-1761.

[20] M. Ernst, H. Geyer, and R. Blickhan, "Spring-Legged Locomotion on Uneven Ground: A Control Approach to Keep The Running Speed Constant," in Proceedings of the International Conference on Climbing and Walking Robots and the Support Technologies for Mobile Machines, 2010, pp. 639-644.

[21] J. W. Hurst, J. E. Chestnutt, and A. a. Rizzi, "Design and philosophy of the BiMASC, a highly dynamic biped," in IEEE International Conference on Robotics and Automation (ICRA), 2007, no. April, pp. 1863-1868.

[22] K. C. Galloway, J. E. Clark, and D. E. Koditschek, "Variable Stiffness Legs for Robust, Efficient, and Stable Dynamic Running," J. Mech. Robot., vol. 5, no. 1, p. 011009, 2013.

[23] J. W. Hurst, J. E. Chestnutt, and A. A. Rizzi, "An actuator with physically variable stiffness for highly dynamic legged locomotion," IEEE Int. Conf. Robot. Autom. 2004. Proceedings. ICRA '04. 2004, vol. 5, pp. 4662-4667, 2004.

[24] K. C. Galloway, J. E. Clark, and D. E. Koditschek, "Design of a MultiDirectional Variable Stiffness Leg for Dynamic Running," in ASME 2007 International Mechanical Engineering Congress and Exposition, 2007, no. August, pp. 73-80.

[25] K. C. Galloway, J. E. Clark, and D. E. Koditschek, "Design of a tunable stiffness composite leg for dynamic locomotion," ASME 2009 Int. Des. Eng. Tech. Conf. Comput. Inf. Eng. Conf., no. August, pp. 215-222, 2009.

[26] R. Ringrose, "Self-stabilizing running," IEEE Int. Conf. Robot. Autom., vol. 1, no. IEEE, 1997.

[27] P. G. Adamczyk, S. H. Collins, and A. D. Kuo, "The advantages of a rolling foot in human walking," J. Exp. Biol., vol. 209, no. 20, pp. 39533963, 2006.

[28] P. G. Adamczyk and A. D. Kuo, "Mechanical and energetic consequences of rolling foot shape in human walking," J. Exp. Biol., vol. 216, no. 14, pp. 2722-2731, 2013.

[29] L. L. Howell and A. Midha, "Parametric Deflection Approximations for Initially Curved, Large-Deflection Beams in Compliant Mechanisms," in Proceedings of the 1996 ASME Mechanisms Conference, 96DETC/MECH-1215, 1995.

[30] A. Rodriguez and M. T. Mason, "Grasp invariance," Int. J. Rob. Res., vol. 31, no. 2, pp. 236-248, Feb. 2012.

[31] K. J. Huang and P. C. Lin, "Rolling SLIP: A model for running locomotion with rolling contact," in 2012 IEEE/ASME International Conference on Advanced Intelligent Mechatronics (AIM), 2012, pp. 2126.

[32] B. R. Whittington and D. G. Thelen, "A Simple Mass-Spring Model With Roller Feet Can Induce the Ground Reactions Observed in Human Walking,” J. Biomech. Eng., vol. 131, no. 1, p. 011013, 2009. 